\title{
Representations of molecular force fields. V. On the equilibrium structure of methane
}

\author{
L. S. Bartell \\ Department of Chemistry, The University of Michigan, Ann Arbor, Michigan 48109
}

Kozo Kuchitsu

Department of Chemistry, Faculty of Science, The University of Tokyo, Bunkyo-ku, Tokyo, Japan (Received 14 July 1977)

\begin{abstract}
The correction from mean $\left(r_{g}\right)$ to equilibrium $\left(r_{e}\right)$ bond lengths in methane is reinvestigated in response to suggestions that the original Kuchitsu-Bartell (KB) corrections were excessive by as much as $0.006 \AA$. It is found that the KB model anharmonic force field with its original parameterization does overcorrect, relative to the new Pulay $a b$ initio force field, but only by $10 \%$ of the original $\left(r_{8}-r_{e}\right)$ corrections, or $0.0022 \AA$ for $\mathrm{CH}_{4}$ and $0.0015 \AA$ for $\mathrm{CD}_{4}$. The $\mathrm{KB}$ model with the more recent MUB-2 nonbonded parameterization gives results in close accord with the $a b$ initio results, supporting the utility of the augmented anharmonic Urey-Bradley representation of force fields. Reported discrepancies between experimental and theoretical methane bond lengths cannot be due solely to the form of or original parameterization of the KB model field.
\end{abstract}

Nearly two decades ago, when excellent equilibrium structures were available for the other simple first-row hydrides, comparable information had not been obtained for methane. Therefore it seemed worthwhile to construct a plausible model force field from which estimates could be made of the vibrational corrections needed to convert spectroscopic $r_{0}$ and electron diffraction $r_{g}$ structures to equilibrium $\left(r_{e}\right)$ structures for $\mathrm{CH}_{4}$ and $\mathrm{CD}_{4}$. Our efforts in this direction ${ }^{1,2}$ were initially regarded as highly speculative but, as time went on, for want of better information, our values became entrenched and often considered as standard. Several years ago, largely through the efforts of Meyer and Pulay,,$^{3,4}$ evidence began to accumulate that the originally proposed equilibrium bond length of $1.085 \AA$ was significantly too short. Several molecular orbital studies were carried out to determine from first principles the structure of minimum energy. The best of these $e^{4}$ suggested a value of $1.0909 \AA$ for $r_{e}$. In addition, $a b$ initio calculations of the quadratic and cubic force field of ethane ${ }^{5}$ showed that the original Kuchitsu-Bartell (KB) model had significant merit in some features but systematic deficiencies in others which could exaggerate the correction $\left(r_{e}-r_{e}\right)$ and, hence, lead to too low an estimation of $r_{e}$. The purpose of this note is to review and update some of the evidence. In concurrent work Pulay et al.$^{6}$ are performing a more comprehensive review.

The original $\mathrm{KB}$ model $^{1}$ adopted a quadratic force field based on the harmonic vibrational frequencies of $\mathrm{CH}_{4}, \mathrm{CD}_{4}$, and $\mathrm{CT}_{4} \cdot{ }^{7}$ This experimental field was decomposed into Urey-Bradley and other components in order to infer the valence and nonbonded contributions and thereby estimate cubic and higher-order terms via Morse and Buckingham empirical force laws. Although the Morse parameter used has proven to be approximately correct, the nonbonded law employed has been found deficient in several respects. Since $\mathrm{H} \cdot \cdots \mathrm{H}$ nonbonded functions in the literature of the time disagreed with each other by more than an order of magnitude it was probably fortuitous that our original guess has turned out to predict certain cubic constants to within a factor of 2. But, for hydrides at least, there is an intrinsic failure of the anharmonic nonbonded model. ${ }^{5,8,9}$ While it more or less correctly accounts for bend and stretch-bend potential constants, it may even give the wrong sign for pure stretch interactions.

To put the anharmonic model into perspective, comparisons are given in Table $I$ of the results of the original $\mathrm{KB}$ work re-expressed in terms of symmetry coordinates, the results of the same model with the recent nonbonded parameters of force field MUB- $2,{ }^{10}$ and the $a b$ initio results of Pulay et al . ${ }^{6}$ Field MUB-2 was formulated in 1974 for theoretical organic conformational analyses by potential energy minimization techniques but it was found to predict with useful accuracy a variety of bend and stretch-bend constants later calculated for ethane. Let force constants be based on the convention

$$
\begin{aligned}
V= & \frac{1}{2} \sum_{i} \sum_{j} f_{i j} \tilde{S}_{i} \tilde{S}_{j} \\
& +\frac{1}{6} \sum_{i} \sum_{j} \sum_{k} \tilde{f}_{i j k} \tilde{S}_{i} \tilde{S}_{j} \tilde{S}_{k}+\cdots,
\end{aligned}
$$

TA BLE I. Methane cubic constants a according to $\mathrm{KB}$ model force field and according to $a b$ initio calculations of Pulay et $a l .^{6}$

\begin{tabular}{llll}
\hline \hline $\begin{array}{l}\text { Cubic } \\
\text { constant }\end{array}$ & $\begin{array}{l}\text { KB model }^{\mathrm{b}} \\
\text { Original } \\
\text { parameters }\end{array}$ & $\begin{array}{l}\text { KB model } \\
\text { MUB-2 } \\
\text { nonbonded }^{\mathrm{c}}\end{array}$ & $\begin{array}{c}\text { Ab initio } \\
\text { Pulay }^{\mathrm{d}}\end{array}$ \\
\hline$f_{111}$ & -19.301 & -17.209 & -15.08 \\
$f_{122}$ & -0.466 & -0.195 & -0.227 \\
$f_{133}$ & -15.740 & -14.958 & -15.47 \\
$f_{134}$ & -0.637 & -0.180 & -0.066 \\
$f_{144}$ & -0.466 & -0.195 & -0.196 \\
\hline
\end{tabular}

aEnergy mđyn $\AA\left(=10^{-18} \mathrm{~J}\right)$, displacements $10^{-8} \mathrm{~cm}$ (stretch), radians (bend). Constants based on curvilinear coordinates. ${ }^{b}$ Reference 1.

${ }^{c}$ Valence stretch anharmonicity from original $\mathrm{KB}$ model. ${ }^{\mathrm{d}}$ Reference 6. 
TABLE II. Comparison of $\left(r_{g}-r_{e}\right)$ corrections calculated for $\mathrm{CH}_{4}$ and $\mathrm{CD}_{4}$ according to various anharmonic model force fields, using the $a b$ initio Pulay anharmonic field ${ }^{a}$ as a reference.

\begin{tabular}{lcc}
\hline \hline & \multicolumn{2}{c}{$\langle r\rangle-\left\langle r_{p}\right\rangle$} \\
\cline { 2 - 3 } Anharmonic force field & $\mathrm{CH}_{4}$ & $\mathrm{CD}_{4}$ \\
\hline Pulay et al. & $0.0022 \AA$ & $(0)$ \\
Original KB & $-0.0026 \AA$ & $-0.0015 \AA$ \\
KB deleting entire nonbonded & & \\
cubic contribution & & \\
KB with MUB-2 nonbonded & $-0.0003 \AA$ & $-0.0003 \AA$ \\
cubic contributions & & \\
\hline
\end{tabular}

${ }^{2}$ Reference 6. All computations in Table II assumed $f_{11}=5.565$ $\mathrm{mdyn} / \check{A}$. From all numbers in Table II is subtracted the quantity $\left(r_{g}-r_{g}\right) \times 0.017$, assuming $\mathrm{CH}$ and $\mathrm{CD}$ bonds behave as Morse oscillators, to compensate for the fact that cubic constants in Ref. 6 were derived using a bond length about $0.0037 \AA$ longer than $r_{e}$.

in which the tilde designates that symmetry coordinates are defined in terms of curvilinear internal coordinates. ${ }^{11,12}$ Cubic constants required for evaluating $\left(r_{g}-r_{e}\right)$ for methane can be written in terms of the $\mathrm{KB}$ model as

$$
\begin{aligned}
& \tilde{f}_{111}=-(3 / 2) a K+\left(2 / r_{e}\right) F_{3}, \\
& \tilde{f}_{122}=\left(r_{e} / 6\right)\left(-2 F^{\prime}+F_{3}\right), \\
& \tilde{f}_{133}=-(3 / 2) a K+\left(2 / 3 r_{e}\right)\left(-F^{\prime}+F+F_{3}\right), \\
& \tilde{f}_{134}=\left(2 F+F_{3}\right) / 3,
\end{aligned}
$$

and

$$
\tilde{f}_{144}=\left(r_{e} / 6\right)\left(-2 F^{\prime}+F_{3}\right) .
$$

For completeness in valence stretch constants the equation

$$
\tilde{f}_{333}=-(3 / 2) a K+\left(1 / r_{e}\right)\left(F-F^{\prime}\right)
$$

is added; it is not needed for $\left(r_{B}-r_{\theta}\right)$. According to the results in Table I the $\mathrm{KB}$ model itself gives a rough and ready account of the anharmonic force field, and a fairly good account when the more modern MUB-2 nonbonded parameters are used (and an optimization based on information subsequent to the 1974 MUB-2 formulation $^{10}$ would do better). The pure stretch defect is still apparent, however.

In view of unpublished suggestions that the $\mathrm{KB}$ correction $\left(r_{g}-r_{e}\right)=0.0221 \check{A}^{13}$ is $0.006 \AA$ too large, making $r_{\varepsilon}$ too low by a like amount, the $\mathrm{KB}$ computations were rechecked. Only trivial errors were found. As explained elsewhere, ${ }^{12}$ the correction $\left(r_{k}-r_{e}\right)$ can be decomposed according to

$$
\left(r_{\varepsilon}-r_{e}\right)=\langle\Delta r\rangle_{C}+\langle\Delta r\rangle_{T}+\delta r_{\text {rot }},
$$

where $\delta r_{\text {rot }}$ is a centrifugal stretch arising from molecular rotation, $\langle\Delta r\rangle_{C}$ is a collection of anharmonic terms each of which is proportional to some (curvilinear) cubic constant $\tilde{f}_{i j k}$ and $\langle\Delta r\rangle_{T}$ is a term in which quadratic constants are propagated into cubic constants of rectilinear displacement coordinates. The dominant physical contribution to $\langle\Delta r\rangle_{T}$ for methane is the mean centrifugal stretch $(\sim 0.003 \AA)$ arising from bending vibrations in which the hydrogen atoms swing over wide arcs with high angular velocities. Inasmuch as $\langle\Delta r\rangle_{T}$ and $\delta r_{\text {rot }}$ depend upon the quadratic potential constants only (which were quite accurately represented by the $\mathrm{KB}$ field) these terms were correctly calculated in the original paper. It is easy to calculate the effect upon $\langle\Delta r\rangle_{C}$ of the difference between a set of cubic constants $\left(\tilde{f}_{a}\right)_{i j k}$ and a set $\left(\tilde{f}_{b}\right)_{i j k}$, by the following equation ${ }^{12}$ :

$$
\begin{aligned}
\left\langle\Delta r_{b}\right\rangle-\left\langle\Delta r_{a}\right\rangle & =\frac{1}{2}\left[\left\langle\tilde{S}_{1}\right\rangle_{b}-\left\langle\tilde{S}_{1}\right\rangle_{a}\right] \\
& =-\left(4 f_{11}\right)^{-1} \sum_{i} \sum_{j}\left(\tilde{f}_{b}-\tilde{f}_{a}\right)_{l i j}\left\langle S_{i} S_{j}\right\rangle,
\end{aligned}
$$

where the mean-square amplitudes $\left\langle S_{i} S_{j}\right\rangle$ are readily computed from the information in Ref. 1 . The results at $0{ }^{\circ} \mathrm{K}$ are described in Table II.

According to Table II the original $\mathrm{KB}$ force field did lead to an excessive $\left(r_{g}-r_{e}\right)$ correction, but only by about $10 \%$, or $0.002 \AA$ (cf, the originally proposed uncertainty of $0.003 \AA$ ). Deleting the cubic nonbonded contributions entirely from the model field is seen to overcorrect the deficiency. Nonbonded cubic constants from MUB- 2 give, by various cancellations, a fortuitously good value for $\left(r_{s}-r_{e}\right)$. Inaccuracies in the original $\mathrm{KB}$ field, then, are insufficient to account for the entire difference between the theoretical and anharmonically corrected experimental values. No doubt part of the discrepancy is in the experimental values, although the diffraction and spectroscopic results are in excellent accord once the correction $\left(r_{s}-r_{0}\right)$ is taken into account. ${ }^{1,2}$ In this regard it should be stressed that the quantity $\left(r_{g}-r_{0}\right)$ can be computed accurately from the quadratic force constants only. ${ }^{14}$ Therefore the spectroscopic correction $\left(r_{0}-r_{e}\right)$ expressible as $\left(r_{0}-r_{g}\right)+\left(r_{g}-r_{e}\right)$ suffers precisely the same distortion from an incorrect anharmonic force field as does the correction $\left(r_{g}-r_{e}\right)$ discussed above.

The original electron diffraction work ${ }^{2}$ with the new corrections of Table $\mathrm{II}^{13}$ suggests the values $( \pm 2 \sigma)$ of $r_{e}(\mathrm{CH})=1.086_{2} \pm 0.0024 \AA$ and $r_{e}(\mathrm{CD})=1.087_{5} \pm 0.0026 \AA$, if the Meyer-Pulay cubic force constants are adopted. Analogous corrections of the spectroscopic results ${ }^{15}$ treated in Ref. 1 yield $1.0865 \check{A}(\mathrm{CH})$ and $1.0862 \AA$ (CD). A more recent electron diffraction study of $\mathrm{CH}_{4}$ by Fink et al. ${ }^{16}$ yields an equilibrium distance of $1.088_{7} \mathrm{~A}$ when corrected similarly. When the experimental errors and theoretical uncertainties in anharmonic constants and in CEFA calculations on hydride bond lengths $(\sim 0.002 \AA)^{17}$ are taken into consideration there does not seem to be a discrepancy requiring explanation. Errors in the original model force field appear to be no larger than originally estimated and little larger than the other uncertainties.

\section{ACKNOWLEDGMENT}

This research was supported by a grant from the National Science Foundation. We are deeply indebted to Dr. P. Pulay for unpublished values of the cubic force constants of methane. 
${ }^{1}$ K. Kuchitsu and L. S. Bartell, J. Chem. Phys. 36, 2470 (1962).

${ }^{2}$ L. S. Bartell, K. Kuchitsu, and R. J. deNeui, J. Chem. Phys. 35, 1211 (1961).

${ }^{3} \mathrm{~W}$. Meyer and P. Pulay, J. Chem. Phys. 56, 2109 (1972).

${ }^{4}$ W. Mever, J. Chem. Phys. 58, 1017 (1973).

${ }^{5}$ L. S. Bartell, S. Fitzwater, and W. J. Hehre, J. Chem. Phys. 63, 4750 (1975).

${ }^{6}$ P. Pulay, W. Meyer, and J. E. Boggs, J. Chem. Phys. (in press). Note that the sign convention for $\tilde{S}_{3}$ and, hence, $\tilde{f}_{333}$ in reference 6 differs from that in reference 1 and the present paper.

${ }^{7}$ L. H. Jones and R. S. McDowell, J. Mol. Spectrosc. 3, 632 (1959).

${ }^{8} \mathrm{~K}$. Kuchitsu and $\mathrm{Y}_{0}$ Morino, Bull. Chem. Soc. Jpn. 38, 805, 814 (1965); Spectrochim. Acta 22, 33 (1966).

${ }^{8}$ Y. Morino, Pure Appl. Chem. 18, 323 (1969).

${ }^{10} \mathrm{~S}$. Fitzwater and L. S. Bartell, J. Am. Chem. Soc. 98, 5107 (1976).

${ }^{11}$ A. R. Hoy, I. M. Mills, and G. Strey, Mol. Phys. 24, 1265 (1972).

${ }^{12}$ L. S. Bartell and S. Fitzwater, J. Chem. Phys. 67, 4168 (1977).

${ }^{13}$ The original results $\left(r_{g}-r_{e}\right)=0.0221 \AA\left(\mathrm{CH}_{4}\right), 0.0164 \AA\left(\mathrm{CD}_{4}\right)$ were based on renormalized first-order wavefunctions. Standard first-order wavefunctions, normalized only through first order, give slightly better results for Morse oscillators and correspond to Eq. (9) of the present text if zeroth-order mean square amplitudes are used. Corresponding values for $\left(r_{\mathrm{g}}-r_{e}\right)$ are $0.0227 \AA\left(\mathrm{CH}_{4}\right)$ and $0.0168 \AA\left(\mathrm{CD}_{4}\right)$.

${ }^{14}$ Y. Morino, K. Kuchitsu, and T. Oka, J. Chem. Phys. 36, 1108 (1962).

${ }^{15}$ R. G. Dickinson, R. T. Dillon, and F. Rasetti, Phys. Rev. 34, 582 (1929); W. H. J. Childs, Proc. R. Soc. London Ser. A 153, 555 (1936); D. R. Boyd, M. W. Thompson, and R. L. Williams. Proc. Roy. Soc. London Ser. A 213, 42 (1952); B. P. Stoicheff, C. Cumming, G. E. St. John, and H. L. Welsh, J. Chem. Phys, 20, 498 (1952); T. Feldman, J. Romanko, and H. L. Welsh, Can. J. Phys. 33, 138 (1955); H. C. Allen and E. K. Plyler, J. Chem. Phys. 26, 972 (1957); B. P. Stoicheff (private communication, 1960); M. A. Thomas and H. L. Welsh, Can. J. Phys. 38, 1291 (1960); A. H. Nielsen and H. H. Nielsen, Phys. Rev. 54, 118 (1938); G. G. Sheppard and H. I. Welsh, J. Mol. Spectrosc. 1, 277 (1957); R. A. Olafson, M. A. Thomas, and H. L. Welsh, Can. J. Phys. 39, 419 (1961).

${ }^{16} \mathrm{M}$. Fink (private communication, 1976).

${ }^{17}$ W. Meyer and P. Rasmus, J. Chem. Phys. 63, 2356 (1975). 